\title{
A New Facility for Fundamental Particle Physics: The High-Intensity Ultracold Neutron Source at the Paul Scherrer Institute
}

\author{
Bernhard Lauss on behalf of the UCN Project Team ${ }^{1}$
}

Paul Scherrer Institute, CH-5232 Villigen-PSI, Switzerland

\begin{abstract}
The fundamental properties of the neutron can be studied in detail using ultracold neutrons (UCN) which can be stored and observed for hundreds of seconds. A new user facility providing ultracold neutrons for fundamental physics research has been constructed at the Paul Scherrer Institute, the PSI UCN source. Assembly of the facility finished in December 2010 with the first production of ultracold neutrons. The layout of the source and its operation are briefly discussed.
\end{abstract}

Keywords: Ultracold neutrons, UCN, Neutron source, ultracold neutron production and transport

PACS: 29.25.Dz.Neutron sources. 28.20.-v Neutron physics

\section{INTRODUCTION}

Neutrons with kinetic energies below $\sim 350 \mathrm{neV}$, corresponding to a few milli-Kelvin, are termed ultracold neutrons (UCN). The material optical potential of certain materials is high enough - due to their large neutron bound-coherent scattering length - that UCN undergo total reflection under all angles of incidence [1]. Hence, UCN can be stored in material bottles for several hundreds of seconds. This provides an excellent possibility to study the fundamental properties of the neutron like its lifetime, its decay properties, or to search for a possible neutron electric dipole moment (nEDM)[1]. nEDM searches (e.g.[2, 3]) probing physics beyond the Standard Model are presently statistics limited and hence are the main drivers for several new high-intensity ultracold neutron sources now being constructed, designed or proposed around the globe [4].

\section{THE ULTRACOLD NEUTRON SOURCE}

Over the last years an ultracold neutron source based on accelerator driven spallation neutron production has been constructed at the Paul Scherrer Institute (PSI), Switzerland.

The concept, experimentally pioneered at PNPI [5] and Los Alamos National Lab [6], is based on

- neutron production via proton induced spallation on lead [7];

- superthermal UCN production in solid ortho-deuterium $\left(\mathrm{s} D_{2}\right)[1]$

- intermediate UCN storage and distribution of UCN on demand to experiments.

The main experimental components covering the neutron production and transport are shown in Fig.1. PSI's $590 \mathrm{MeV}$ proton beam with up to $2.4 \mathrm{~mA}$ beam current is impinging on a heavy water cooled canneloni-type lead spallation target (see (1) in Fig.1) [7]. UCN operation is limited to 1\% duty cycle. This means a full proton beam kick of up to $8 \mathrm{~s}$ onto the target is followed by a minimum $800 \mathrm{~s}$ waiting period. Spallation neutrons are thermalized in the surrounding heavy water tank (2). $50 \mathrm{~cm}$ close to the spallation target inside a pure AlMg3 container (3) a 30 liter ortho-deuterium crystal is kept at a temperature of $5 \mathrm{~K}$. It serves to produce first cold and finally ultracold neutrons via downscattering $[1,4,9]$

The UCN production rate strongly depends on the deuterium temperature and spin $[8,9]$ strongly favoring solid ortho-deuterium as production medium. Some UCN can then be emitted into the vacuum on top of the crystal, where they get a $102 \mathrm{neV}$ boost from the $\mathrm{s} D_{2}$ material potential at the crystal surface. Via a vertical UCN guide (4) they can reach the UCN storage volume (5) coated with diamond-like carbon which has a high material optical potential of

\footnotetext{
${ }^{1}$ The members of the PSI UCN Project Team are listed at http://ucn.web.psi.ch.
}

19th Particles and Nuclei International Conference (PANIC11)

AIP Conf. Proc. 1441, 576-578 (2012); doi: 10.1063/1.3700622

(C) 2012 American Institute of Physics 978-0-7354-1035-0/\$30.00 


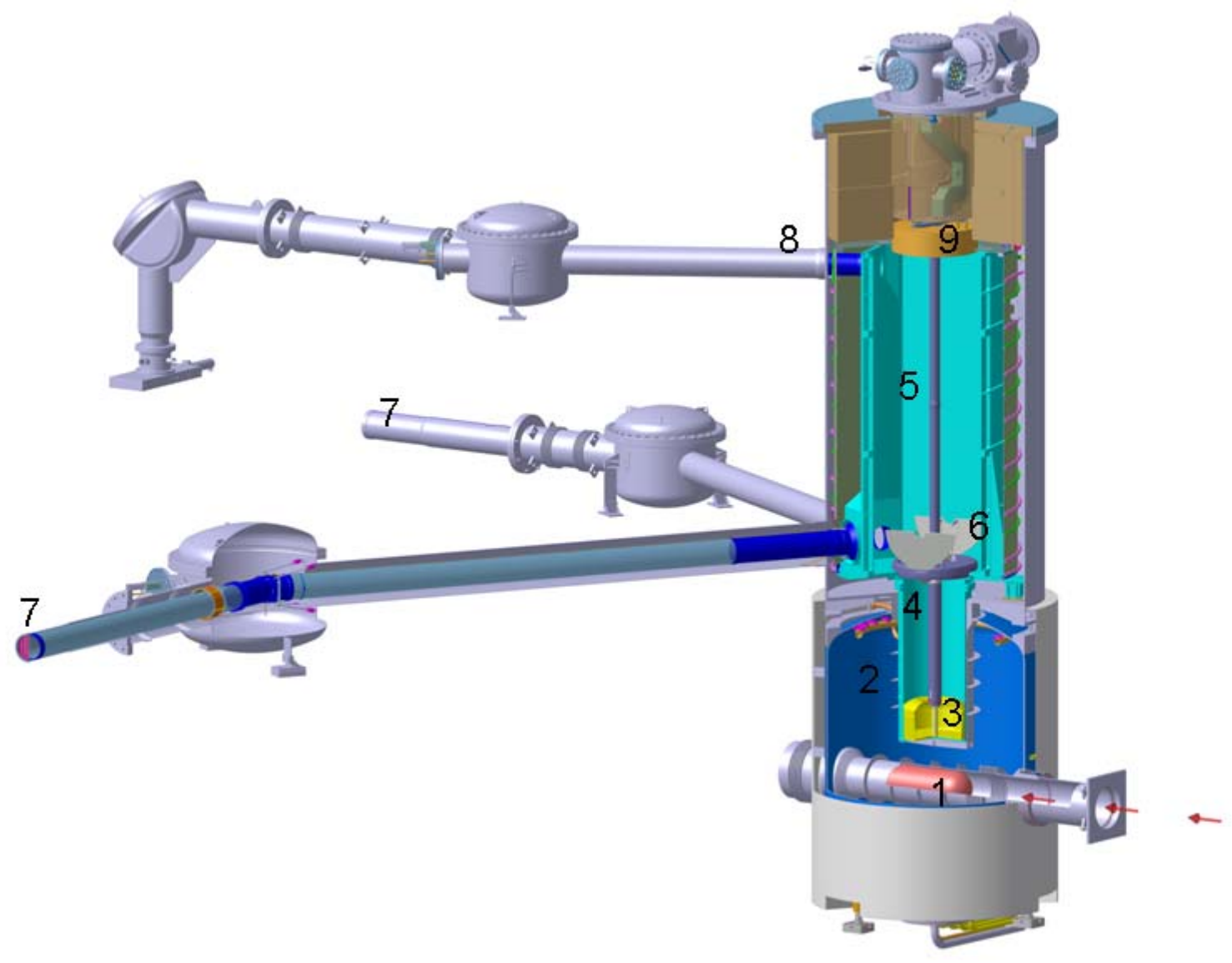

FIGURE 1. Cut view of the neutron production, storage and guiding system of the PSI UCN source inside a $7 \mathrm{~m}$ high vacuum tank. The indicated components are described in the text.

about $235 \mathrm{neV}$ and a very low neutron loss per bounce. After the beam kick the main UCN shutter (6) is rapidly closed and the produced UCN are stored inside the volume. Three UCN guides $(7,8)$ lead through the radiation shielding to experimental areas. Small shutters can open and close these guides at the storage volume exits. In order to provide optimal UCN transmission over more than $8 \mathrm{~m}$ of tubes passing through the radiation shielding, most of the guides are made of $180 \mathrm{~mm}$ inner diameter Duran glass tubes with $1 \mathrm{~nm}$ surface roughness and sputter-coated with $500 \mathrm{~nm}$ thick NiMo on the inside. A $80 \mathrm{~cm}$ long polished steel guide connects the glass guides at room temperature to the UCN storage volume which is operating at $80 \mathrm{~K}$. Radiation protection requires a $30^{\circ}$ bend made from polished stainless steel to prevent direct sight onto the storage volume. On top of the UCN storage volume a large, activated carbon loaded cryo-pump is the coldest spot of the source vacuum in order to capture eventual residual gas atoms and prevent them from condensing on the $\mathrm{s} D_{2}$ container. Pictures of the components can be found in [10].

Several important components are not shown on the figure. Most prominently the cryo-system necessary for the production of the ortho-deuterium crystal. The present $\mathrm{s} D_{2}$ production procedure [11] uses $30 \mathrm{~m}^{3}$ pure $D_{2}$ gas. The gas is frozen into the 'condenser' volume. Then it is liquefied and transported to the nearby 'para-otho-converter' filled with a paramagnetic chromium-oxide $\left(\right.$ Oxisorb $\left.^{\circledR}\right)$ and left boiling at $19 \mathrm{~K}$ for several hours. Raman spectroscopy of the rotational transitions in $D_{2}$ on extracted gas samples shows an ortho-deuterium concentration of $97 \pm 2 \%$ as expected for equilibrium conditions at $19 \mathrm{~K}$. Finally, the ortho- $D_{2}$ is slowly frozen inside the crystal container. This process takes several days and, as a very important part of the source setup, still needs to be optimized.

Final assembly of all parts and commissioning of the PSI source finished in 2010. December 16, 2010 saw the first UCN production during testing the radiological and cryogenic safety. Fig. 2 shows one of the first production fills with 


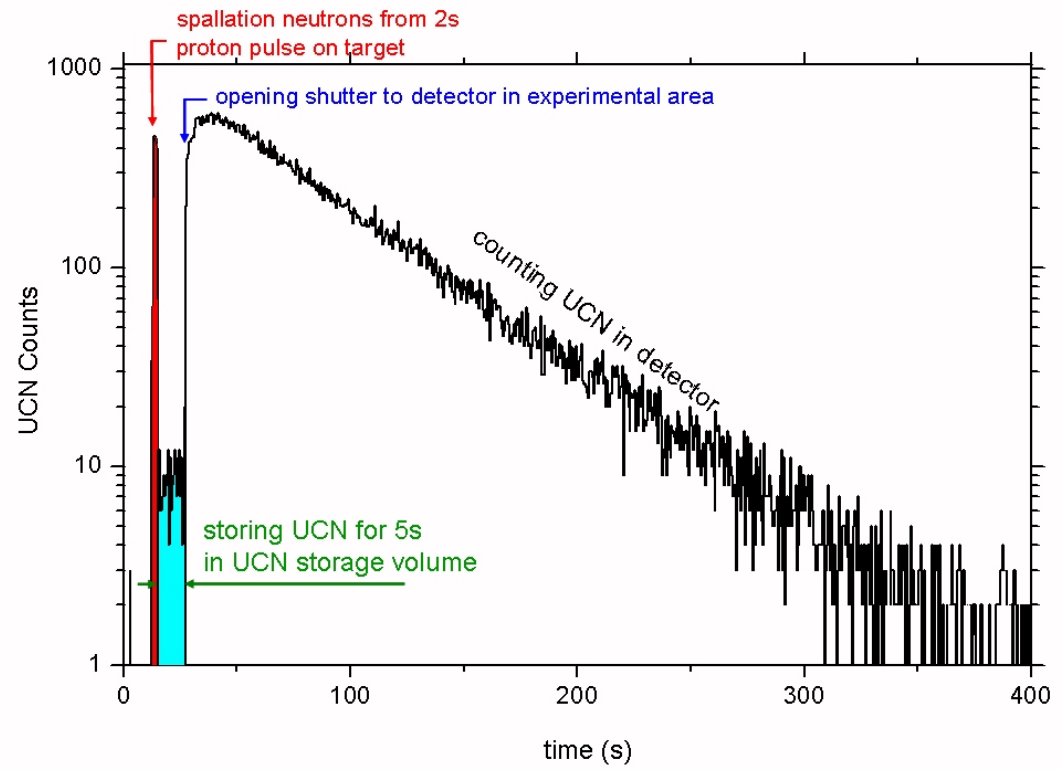

FIGURE 2. First UCN in December 2010: UCN counts observed in a Cascade-U detector located at the end of the UCN guide in area West, plotted versus time (seconds). The structure in the count rate reflects the initial proton beam kick onto the spallation target of $2 \mathrm{~s}$ which produce a neutron background in the detector. After the beam kick the UCN storage volume is closed and the UCN are stored for $5 \mathrm{~s}$. A few percent of the UCN leak during that time through the guide UCN shutter. After $5 \mathrm{~s}$ the guide shutter towards the experimental area is opened and the UCN can arrive at the detector. The observed count rate then reflects the exponential emptying of the UCN storage volume into the detector. UCN can then be observed over a few hundred seconds.

a count-rate structure as expected. Beam kicks of up to $8 \mathrm{~s}$ length and full beam power were tested.

Operation approval of the Swiss Federal Authorities was received on June 27, 2011. The PSI UCN source is now starting up and we are continuously improving the source performance. The experimental search for a nEDM is starting in area South with an installed apparatus [2]. The UCN user facility at PSI is now open for experiment proposals.

\section{ACKNOWLEDGMENTS}

Cordial thanks to the more than two hundred colleagues contributing to the UCN source project at the Paul Scherrer Institute who are indispensable for the realization of this project. Support of our colleagues at PF2 - ILL and the Mainz TRIGA UCN Source during component testing is acknowledged. PNPI contributed in the early planning of the project.

\section{REFERENCES}

1. R. Golub, D. Richardson, S.K. Lamoreaux, Ultra-Cold Neutrons, Adam Hilger, Bristol, Philadelphia and New York, 1991.

2. K. Kirch et al., these proceedings.

3. C.A. Baker et al., Phys. Rev. Lett. 97, 131801(2006).

4. K. Kirch, B. Lauss, P. Schmidt-Wellenburg, G. Zsigmond, Nucl. Phys. News 20, 17 (2010).

5. A.P. Serebrov et al., JETP Lett. 66, 802 (1997).

6. C.L. Morris et al., Phys. Rev. Lett. 89, 27250 (2002).

7. M. Wohlmuther, G. Heidenreich, Nucl. Instr. Meth. A 564, 51(2006).

8. C.-Y. Liu et al., Phys. Rev. B 62, R3581 (2000).

9. F. Atchison et al., Phys. Rev. C 71, 05460 (2005).

10. B. Lauss, Proceedings of the International Nuclear Physics Conference - INPC-2010, July 4 - 9, 2010, Vancouver; nucl-ex:1011.3993

11. A. Anghel et al., Proc. of the 10th IIR International Conference Cryogenics 2008, 04/21 - 04/25, 2008, Prague, Czech Republic. 